nephron

Practice
Nephron 2016;134:10-13

DOI: $10.1159 / 000445080$
Received: January 16, 2016

Accepted after revision: February 27, 2016

Published online: March 19, 2016

\title{
Mineralocorticoid Receptor Antagonism: A Promising Therapeutic Approach to Treat Ischemic AKI
}

\author{
Jonatan Barrera-Chimal ${ }^{a, c}$ Norma A. Bobadilla ${ }^{c}$ Frédéric Jaisser ${ }^{a, b}$ \\ aINSERM, UMRS 1138, Team 1, Centre de Recherche des Cordeliers, Pierre et Marie Curie University, Paris Descartes

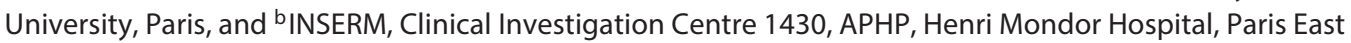 \\ University, Creteil, France; ${ }^{C}$ Molecular Physiology Unit, Instituto de Investigaciones Biomédicas, Universidad \\ Nacional Autónoma de México and Nephrology Department, Instituto Nacional de Ciencias Médicas y Nutrición \\ Salvador Zubirán, Mexico City, Mexico
}

\section{Key Words}

Acute renal injury Aldosterone $\cdot$ Endothelial nitric oxide synthase $\cdot$ Endothelin $1 \cdot$ Nitrix oxide

\begin{abstract}
Acute kidney injury (AKI) is a common complication in hospitalized patients. One of the leading causes of AKI is renal ischemia/reperfusion (IR). In spite of all the progress made in acquiring knowledge about the mechanisms involved in AKI, no pharmacologic approach has yet become successful in clinical trials. Recent evidence suggests that mineralocorticoid receptor (MR) antagonism may be a useful strategy to prevent or treat AKI induced by IR. Here, we summarize the experimental work that supports MR antagonism as a potential approach to treat this disease. We also review the evidence that identifies a critical mechanism participating in the sustained vasoconstriction during kidney IR and uncovers that this mechanism is targeted by MR antagonists, thus explaining their beneficial effects.

(c) 2016 S. Karger AG, Basel
\end{abstract}

Contribution from the Special Symposium to celebrate the contributions of David G. Warnock, MD to Academic Nephrology, The University of Alabama at Birmingham, Division of Nephrology, November 9, 2015.

\section{KARGER}

๑) 2016 S. Karger AG, Basel

E-Mail karger@karger.com

www.karger.com/nef
Acute kidney injury (AKI) is a frequent complication in hospitalized patients with higher incidence rates in intensive care unit patients. It is associated with unfavorable outcomes such as increased short- and long-term mortality rates, longer hospital stay, cardiovascular complications and chronic kidney disease (CKD) development [1]. Since many clinical situations can lead to a partial or complete reduction in the renal blood flow, an important cause of AKI is renal ischemia/reperfusion (IR) [2]. Ischemic injury is a complex pathophysiological entity that involves several alterations in the kidney. The reduction in the oxygen delivery leads to endothelial cell death, capillary loss, increase in capillary permeability, tubular cell death and to the activation of the surviving endothelial cells. Especially, direct injury in S2 and S3 segment of the proximal tubule epithelium due to reduced oxygen delivery and reactive oxygen species (ROS) generation during reperfusion take place [3]. In addition, an imbalance in vasoactive factors occurs and favors an increase in vasoconstriction, thus maintaining and prolonging the initial reduction in the renal blood flow [4]. All these alterations facilitate the initiation of an inflammatory process that will propagate the initial injury [4]. Unfortunately, even though the knowledge of AKI mechanisms is increasing, there is no effective pharmacological approach to treat or prevent AKI that has been successful in clinical trials.

Dr. Frédéric Jaisser

INSERM U1138, Centre de Recherche de Cordeliers, Team 1 Pierre et Marie Curie University, Paris Descartes University 15 rue de l'Ecole de Médecine, FR-75006 Paris (France)

E-Mail frederic.jaisser@inserm.fr 


\section{MR Antagonism Protects against Ischemic AKI in Rodents}

During the past decade, evidence indicating that mineralocorticoid receptor (MR) antagonism (MRA) may be a useful strategy to protect against AKI has been accumulating. In 2007, a study by Mejía-Vilet et al. [5] showed that in a rat model of bilateral renal IR, the prophylactic administration of spironolactone (Spiro) prevented the acute renal dysfunction and tubular injury induced by IR. Spiro beneficial effect was associated with the preservation of a normal renal blood flow and reduction in oxidative stress. In this study, we suggested that the prevention of the hypo-perfusion induced by IR was mediated by acting on the eNOS/NO pathway, since the levels of urinary nitrates and nitrites were restored in Spiro treated rats. Moreover, this group presented increased eNOS expression and reduced phosphorylation in the Threonine 495 of eNOS, a key residue that when it is phosphorylated, it prevents eNOS from producing NO. In a subsequent study by Sánchez-Pozos et al. [6], it was demonstrated that Spiro is also able to treat kidney IR injury efficiently when administered immediately or up to $3 \mathrm{~h}$ after reperfusion. The benefit of Spiro at $6 \mathrm{~h}$ after reperfusion was partial and was lost when administered after longer periods of time. The renal hypo-perfusion was also prevented by Spiro treatment, thus suggesting a key role for MR activation in mediating vasoconstriction during renal IR. This study reported that renal vasoconstriction was associated with a reduction in the expression of the endothelin (ET)-B receptor, which mediates vasodilator effects in endothelial cells, and an increase in ET-A receptor, that mainly mediates vasoconstrictor effects. This imbalance in ET-A/ET-B receptor expression was not observed in rats receiving Spiro, thus gaining some insights into the possible mechanisms responsible for the beneficial effects of MR inhibition during kidney IR.

\section{Aldosterone as a Key Mediator of AKI}

To further explore if activation of MR by aldosterone is responsible for the deleterious effects observed during IR, we studied rats that underwent adrenalectomy and dexamethasone supplementation and were subjected to renal IR. These rats were efficiently protected from all the alterations induced by the ischemic insult in a similar way as Spiro administration does, leading to the conclusion that aldosterone plays a key role in the vasoconstriction and therefore in the deleterious effects of renal IR [7].

\section{Non-Steroidal MRA Protects against AKI}

Although the blockade of MR seems to be a promising therapeutic approach to treat AKI, the use of Spiro in renal ischemia is restricted due to its potassium-sparing effects, especially in patients with compromised renal function. In this sense, it is important to mention about the recent development of novel non-steroidal MR antagonists such as BR- 4628 or finerenone that retain the beneficial effects of previously existing MR antagonists at a lower administration dose and with a lower risk of hyperkalemia $[8,9]$. Indeed, finerenone has reduced the deleterious effects on plasma potassium levels as compared to Spiro in patients with heart failure and mild renal disease in spite of similar benefits in B-type natriuretic peptide levels [10].

In a recent study [11], we tested the efficacy of a novel, non-steroidal MRA, BR-4628, to protect against renal injury induced by IR. We showed that BR- 4628 administration prevents and treats renal dysfunction, tubular injury and oxidative stress induced by IR. In order to provide new insights into the mechanism of kidney protection conferred by MRA, we studied the effects of renal IR and MR inhibition on the ET-1/ET-A/ET-B receptor pathway. We identified a critical mechanism participating in the sustained vasoconstriction during kidney IR and provide evidence that this mechanism is targeted by MRA, thus explaining the beneficial effects of MR antagonists. We demonstrated that the protective effect of BR-4628 on renal function, hypo-perfusion and tubular injury was lost when a selective ET-B antagonist was co-administered, pointing out the importance of the ET-B receptor in mediating the protection conferred by MR antagonists. It was previously reported that in pulmonary endothelial cells, aldosterone induces an increase in ROS generation that in turn allows a sulfenic modification of the Cys- 405 residue in the ET-B receptor avoiding the binding to heterotrimeric G-protein beta-gamma-subunits, which signal to AKT [12]. Therefore, AKT reduction results in decreased eNOS activating phosphorylation. We therefore analyzed if this modification also happened in the kidney during IR. We showed for the first time in vivo and confirmed that in the ischemic kidney, the ET-B receptor undergoes a sulfenic acid modification of a critical cysteine residue, decreasing eNOS activation and affecting renal hemodynamics. The post-translational modification of the ET-B receptor and its consequences on eNOS signaling and NO production were efficiently prevented by MRA, suggesting that sulfenic modification of the Cys405 residue in the ET-B occurs as a consequence of in- 
Fig. 1. Mechanisms contributing to sustained vasoconstriction after renal IR. Renal IR increases aldosterone and ET-1 levels. Activation of MR by aldosterone may promote an augmentation in the expression of ET-A receptor (mediates ET-1 induced vasoconstriction) and ROS; effects blunted by MRA. ROS generation in turn induces a sulfenic acid modification in ET-B receptor, which accounts for the reduced activation of eNOS and NO production. The reduction in the bioavailability of NO, together with the increased expression of ET-A receptor shifts the balance toward vasoconstriction. The sustained vasoconstriction induces hypoxia in the kidney tissue leading to tubular injury. Injured tubules may go through maladaptive repair and produce CKD development.

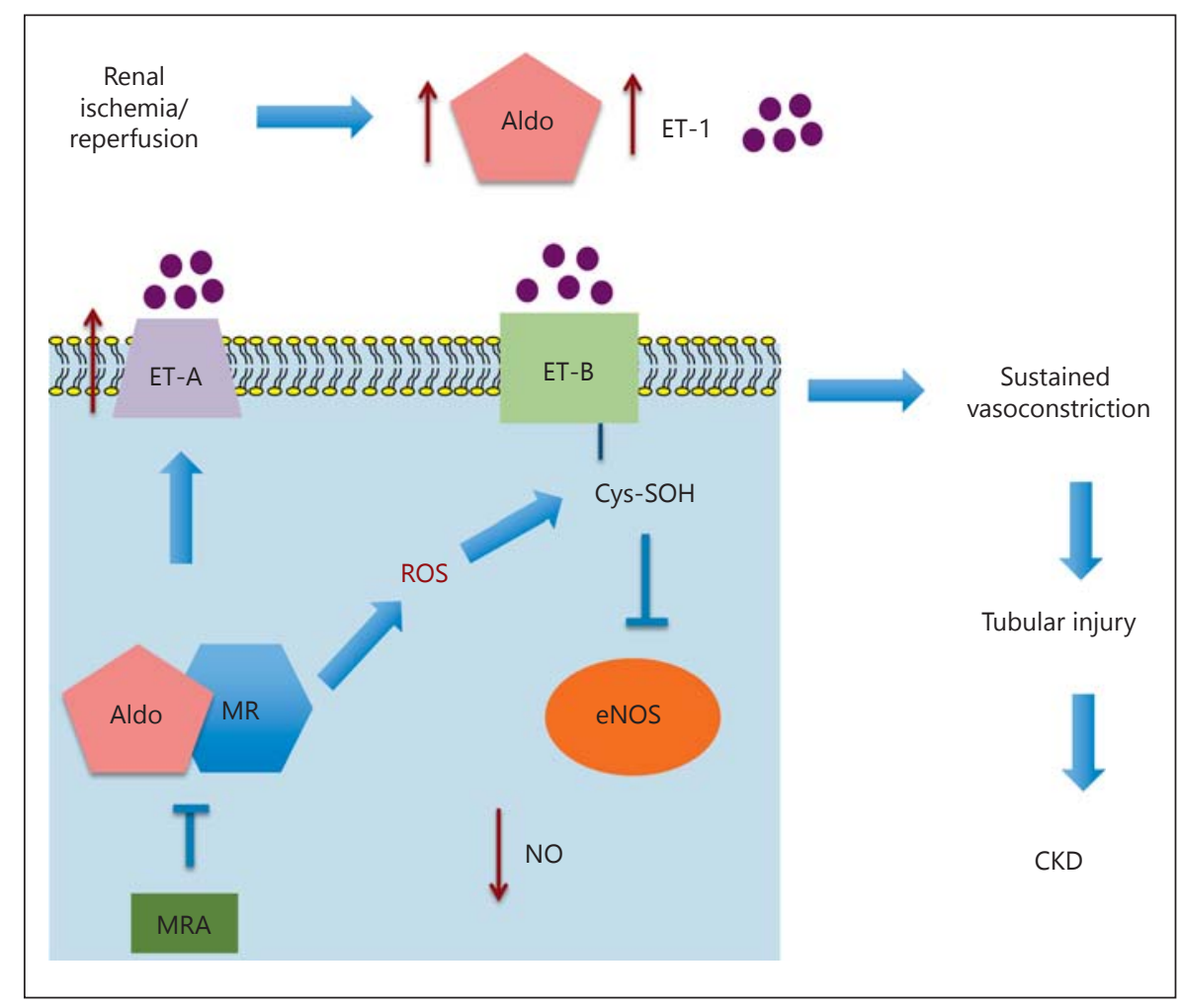

creased oxygen reactive species after IR. The fact that ET-B receptor is inactivated during kidney IR may promote vasoconstriction by 2 different pathways: first, by a reduction of eNOS activation due to ET-B receptor sulfenic modification; second by shifting the balance to ET-1 mediated vasoconstriction by ET-A receptor (fig. 1). As commented by Juncos and Juncos [13], the fact that MRA acts through different mechanisms in order to prevent vasoconstriction makes $\mathrm{MR}$ a target that can be effective in treating AKI.

\section{MRA Prevents the Transition from AKI to CKD}

MRA holds promise not only to prevent the acute consequences of IR but also to avoid the progression to CKD induced by renal IR. Previously we showed [14] that a single episode of bilateral renal IR in rats induced CKD after a 9-month follow-up. The main alterations observed were renal dysfunction, proteinuria, extensive tubular dilation, glomerular hypertrophy and sclerosis and interstitial fibrosis development. Importantly, prophylactic MR inhibition for a few days before AKI was able to prevent the development of all these alterations.

\section{Spironolactone Reduces Oxidative Stress in Kidney Transplantation}

In support of all these experimental evidence showing a beneficial effect of MRA in ischemic kidney injury, we performed a pilot study to analyze the effect of Spiro administration in preventing adverse outcomes after living-kidney transplantation. Renal function was already well preserved in these patients receiving livingdonor grafts, but it was not improved compared with untreated renal transplant patients. Interestingly, the patients receiving Spiro presented lesser levels of urinary hydrogen peroxide, a marker of oxidative stress [15]. Whether MRA may be beneficial in patients transplanted with grafts with expanded criteria that are more susceptible to IR injury and delayed graft function will be tested in the ongoing EPURE TRANSPLANT clinical trial (NCT02490904).

In conclusion, MRA, especially with novel non-steroidal antagonists, is a promising therapeutic strategy to prevent or limit kidney injury induced by IR both in acute setting and in AKI-mediated CKD. The current evidence calls for future research to test MRA efficiency in patients at high risk of developing AKI such as those in intensive care units, kidney transplant and cardiac surgery patients. 


\section{Acknowledgments}

This work was supported by grants from Institut National de la Santé et de la Recherche Médicale and from the ECOS-CONACyT exchange program (ECOS M13S03/CONACYT229524). J.B.-C. was supported by a postdoctoral fellowship from the French Society of Nephrology (SPF2012FDR_SN_FRM_Barrera Chimal) and the French Foundation for Medical Research (SPF20130526725).

\section{Disclosure Statement}

Frédéric Jaisser received a research grant from Bayer.

\section{References}

$\checkmark 1$ Lameire NH, Bagga A, Cruz D, De Maeseneer J, Endre Z, Kellum JA, Liu KD, Mehta RL, Pannu N, Van Biesen W, Vanholder R: Acute kidney injury: an increasing global concern. Lancet 2013;382:170-179.

- 2 Matejovic M, Ince C, Chawla LS, Blantz R, Molitoris BA, Rosner MH, Okusa MD, Kellum JA, Ronco C; ADQI XIII Work Group: Renal hemodynamics in AKI: in search of new treatment targets. J Am Soc Nephrol 2016;27:49-58.

3 Sharfuddin AA, Molitoris BA: Pathophysiology of ischemic acute kidney injury. Nat Rev Nephrol 2011;7:189-200.

-4 Bonventre JV, Yang L: Cellular pathophysiology of ischemic acute kidney injury. J Clin Invest 2011;121:4210-4221.

5 Mejía-Vilet JM, Ramírez V, Cruz C, Uribe N, Gamba G, Bobadilla NA: Renal ischemia-reperfusion injury is prevented by the mineralocorticoid receptor blocker spironolactone. Am J Physiol Renal Physiol 2007;293:F78F86.

6 Sánchez-Pozos K, Barrera-Chimal J, GarzónMuvdi J, Pérez-Villalva R, Rodríguez-Romo R, Cruz C, Gamba G, Bobadilla NA: Recovery from ischemic acute kidney injury by spironolactone administration. Nephrol Dial Transplant 2012;27:3160-3169.
Ramírez V, Trujillo J, Valdes R, Uribe N, Cruz C, Gamba G, Bobadilla NA: Adrenalectomy prevents renal ischemia-reperfusion injury. Am J Physiol Renal Physiol 2009;297:F932F942.

8 Fagart J, Hillisch A, Huyet J, Bärfacker L, Fay M, Pleiss U, Pook E, Schäfer S, Rafestin-Oblin ME, Kolkhof P: A new mode of mineralocorticoid receptor antagonism by a potent and selective nonsteroidal molecule. J Biol Chem 2010;285:29932-29940.

-9 Kolkhof P, Nowack C, Eitner F: Nonsteroidal antagonists of the mineralocorticoid receptor. Curr Opin Nephrol Hypertens 2015;24: 417-424.

10 Pitt B, Anker SD, Böhm M, Gheorghiade M, Køber L, Krum H, Maggioni AP, Ponikowski P, Voors AA, Zannad F, Nowack C, Kim SY, Pieper A, Kimmeskamp-Kirschbaum N, Filippatos G: Rationale and design of MinerAlocorticoid Receptor antagonist Tolerability Study-Heart Failure (ARTS-HF): a randomized study of finerenone vs. eplerenone in patients who have worsening chronic heart failure with diabetes and/or chronic kidney disease. Eur J Heart Fail 2015;17:224-232.
11 Barrera-Chimal J, Prince S, Fadel F, El Moghrabi S, Warnock DG, Kolkhof P, Jaisser F: Sulfenic acid modification of endothelin B receptor is responsible for the benefit of a nonsteroidal mineralocorticoid receptor antagonist in renal ischemia. J Am Soc Nephrol 2016;27:398-404.

12 Maron BA, Zhang YY, White K, Chan SY, Handy DE, Mahoney CE, Loscalzo J, Leopold JA: Aldosterone inactivates the endothelin-B receptor via a cysteinyl thiol redox switch to decrease pulmonary endothelial nitric oxide levels and modulate pulmonary arterial hypertension. Circulation 2012;126:963-974.

13 Juncos LA, Juncos LI: Mineralocorticoid receptor antagonism in AKI: a new hope? J Am Soc Nephrol 2016;27:335-337.

14 Barrera-Chimal J, Pérez-Villalva R, Rodríguez-Romo R, Reyna J, Uribe N, Gamba G, Bobadilla NA: Spironolactone prevents chronic kidney disease caused by ischemic acute kidney injury. Kidney Int 2013;83:93-103.

15 Ojeda-Cervantes M, Barrera-Chimal J, Alberú J, Pérez-Villalva R, Morales-Buenrostro LE, Bobadilla NA: Mineralocorticoid receptor blockade reduced oxidative stress in renal transplant recipients: a double-blind, randomized pilot study. Am J Nephrol 2013;37: 481-490. 